\title{
Indirect Dark Matter searches with Fermi
}

\author{
Johann Cohen-Tanugi ${ }^{* \dagger}$
}

The Fermi Gamma-Ray Space Telescope mission, formerly known as GLAST, is the next generation satellite for high-energy gamma-ray astronomy. It was successfully launched on June 11th 2008, and is currently orbiting around the Earth for a planned one-year observation of the full sky. The Large Area Telescope (LAT), its main instrument, with a wide field of view ( $>2 \mathrm{sr}$ ), a large effective area $\left(>8000 \mathrm{~cm}^{2}\right.$ at $\left.1 \mathrm{GeV}\right)$, sub-arcminute source localization, a large energy range $(20 \mathrm{MeV}-300 \mathrm{GeV}$ ) and a good energy resolution (close to $8 \%$ at $1 \mathrm{GeV}$ ), has excellent potential to either discover or to constrain a dark matter signal. This contribution reports on current performance of the LAT and reviews the complementary searches for signatures of particle dark matter that the LAT team is currently pursuing.

Identification of dark matter 2008

August 18-22, 2008

Stockholm, Sweden

* Speaker.

${ }^{\dagger}$ on behalf of the Fermi-LAT Collaboration 


\section{Introduction}

Though compelling evidence converge toward a picture of the Universe where about $20 \%$ of the mass is non baryonic and seemingly manifests itself only through gravitation [1], the nature and properties of this Dark Matter component remains an outstanding question for modern physics. Several plausible extentions to the Standard Model of Particle physics contain a Dark Matter candidate, with predictions to be tested experimentally. Among them, a much studied scenario, arising for instance in supersymmetric or universal extra-dimensional extensions, consists of a weakly interacting massive particle (WIMP) that could pair-annihilate into various channels giving rise to neutrinos, hadrons, leptons, and photons as final products. These photons can in principle fall in the energy band of space or ground based gamma-ray detectors, thus allowing for the indirect detection of Dark Matter in the gamma-ray sky.

Quite irrespective of the underlying theoretical model, the gamma-ray continuum flux from WIMP annihilation at a given photon energy $E_{\gamma}$ from a direction that forms an angle $\psi$ between the direction of the Galactic Center and that of observation is given by [2]:

$$
\phi_{\mathrm{WIMP}}\left(E_{\gamma}, \psi\right)=\frac{1}{4 \pi} \frac{<\sigma_{\mathrm{ann}} v>}{2 m_{\mathrm{WIMP}}^{2}} \sum \frac{d N_{\gamma}^{f}}{d E_{\gamma}} B_{f} \int_{\mathrm{los}} \rho(l)^{2} d l(\psi) .
$$

The particle physics model enters through the WIMP mass $m_{\text {WIMP }}$, the total mean annihilation cross-section $\sigma_{\text {ann }}$ multiplied by the relative velocity of the particles (in the limit of $v \rightarrow 0$ ), and the sum of all the photon yields $d N_{\gamma}^{f} / d E_{\gamma}$ for each annihilation channel weighted by the corresponding branching ratio $B_{f}$. For Majorana fermion WIMPs light fermions are suppressed so that the dominant fermionic annihilation products will be $b \bar{b}, t \bar{t}, W^{+} W^{-}$, and $\tau^{+} \tau^{-}$. Except for the $\tau^{+} \tau^{-}$ channel, the photon yields are rather similar [3] though internal bremmstrahlung can significantly alter the corresponding spectrum [4]. In the special case of the $2 \gamma$ final state, a spectral line is centered on energy $E_{\gamma}=m_{\text {WIMP }}$. This process is loop suppressed with a branching fraction of usually $10^{-3}$ to $10^{-4}$ (however, see also [5]). The spatial distribution of the WIMPs is considered in the integral along the line of sight (los) of the assumed density squared $\rho(l)^{2}$.

\section{First light with the Fermi mission and the LAT performance}

The Fermi Gamma-Ray Space Telescope (hereafter "Fermi") [6,7], which is part of NASA's office of Space and Science strategic plan, is an international space mission that studies cosmic $\gamma$-rays in the energy range $20 \mathrm{MeV}-\geq 300 \mathrm{GeV}$. This mission is the result of an international collaboration between the astrophysics and particle physics communities, including institutions in the USA, Japan, France, Germany, Italy and Sweden. The main instrument on Fermi is the Large Area Telescope (LAT) complemented by a dedicated instrument for the detection of gamma-ray bursts (the Gamma-ray burst monitor, GBM).

The baseline of the Fermi LAT detector is modular, consisting of a $4 \times 4$ array of identical towers, as shown in Figure 1 . Each $40 \times 40 \mathrm{~cm}^{2}$ tower comprises a tracker, a calorimeter and a data acquisition module. The tracking detector consists of 18 xy layers of silicon strip detectors. This detector technology has a long and successful history of application in accelerator-based highenergy physics. It is well-matched to the requirements of high detection efficiency (>99\%), 
excellent position resolution $(<60 \mu \mathrm{m})$, large signal/noise $(>20)$, negligible cross-talk, and ease of trigger and readout. Compared to its predecessor EGRET [8], the LAT improves on point source sensitivity by at least a factor of 30, the energy range is extended by a factor 10 and the energy $(8 \%$ at $10 \mathrm{GeV})$ and angular resolutions $\left(\mathrm{PSF}_{68 \%}=0.1^{\circ}\right.$ at $\left.10 \mathrm{GeV}\right)$ are improved by a factor of at least two. Furthermore, the choice of silicon tracking detectors instead of the spark-chambers used in EGRET reduces the dead-time by more than three orders of magnitude.

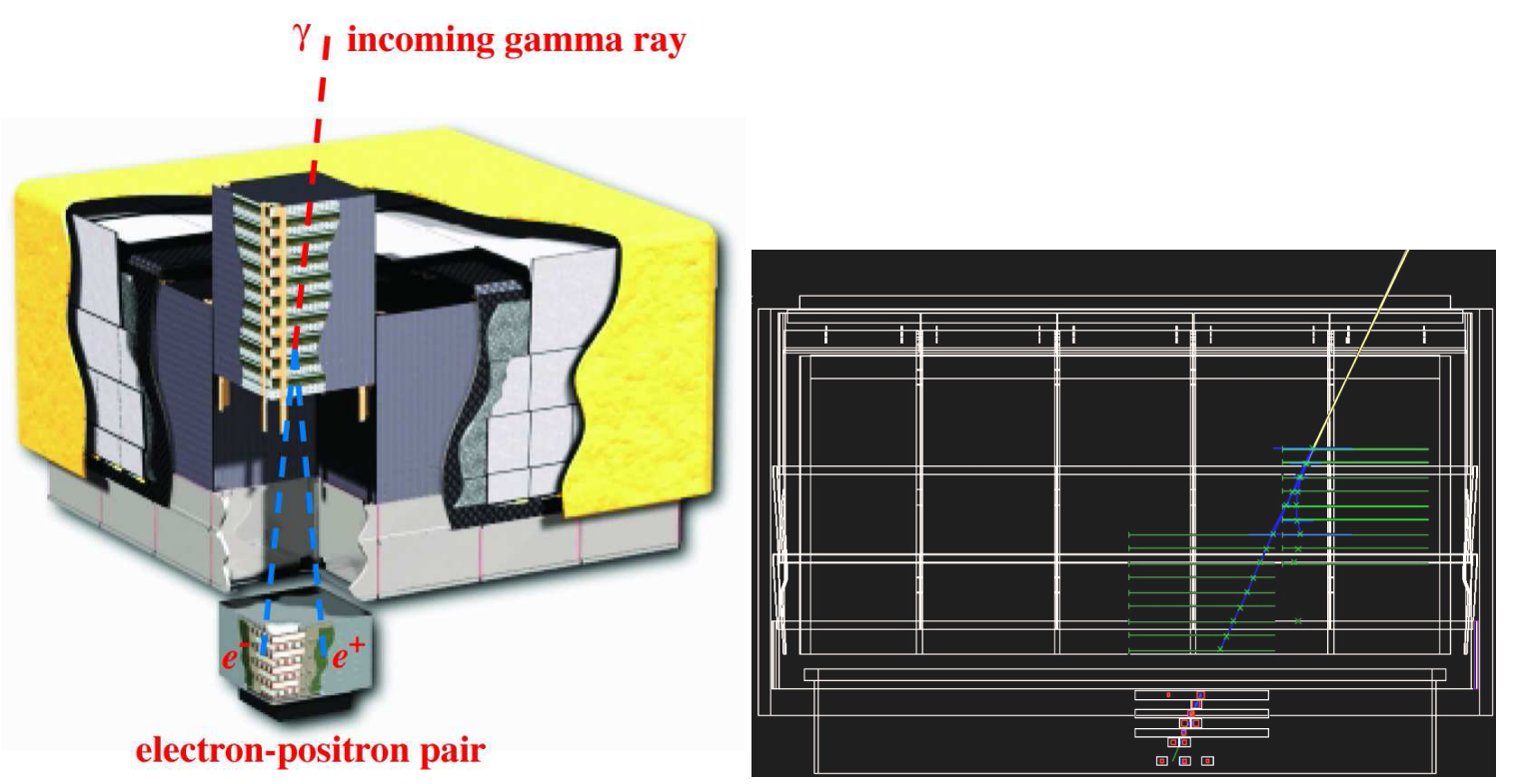

Figure 1: Left : Schematic diagram of the Large Area Telescope. The telescope's dimensions are $1.8 \mathrm{~m}$ $\times 1.8 \mathrm{~m} \times 0.72 \mathrm{~m}$. The power required and the mass are $650 \mathrm{~W}$ and $2,789 \mathrm{~kg}$, respectively. Right : event display of a candidate gamma-ray, its direction (yellow) being determined by its supposed daughter tracks (blue), reconstructed from the cluster of strips (green) where they intersect the tracker silicon planes. The deposited energy in the calorimeter (red) allow for the determination of the incident energy.

After its launch on June 11th 2008, Fermi underwent a 60-day period of calibration and commissioning, during which safe command of the spacecraft and instruments and quality of the data were asserted. The LAT was shown to have suffered no harm from the launch, and to perform as expected and to closely match Monte Carlo simulations. Figure 2 compares such a simulation with te EGRET legacy.

Thanks to its unprecedented sensitivity, very good energy resolution, and large field of view ( $\sim 2.4 \mathrm{sr}$ ), the LAT is able to function nominally in sky-survey mode, where $20 \%$ of the sky is seen at each instant (see Figure 3). As a consequence, the scientific topics addressed by the LAT cover a very large domain of high-energy astrophysics: (i) what is the nature of the unidentified sources and the origins of the diffuse emission revealed by EGRET? (ii) What are the mechanisms of particle acceleration operating in celestial sources, particularly in active galactic nuclei, pulsars, supernovae remnants, and the Sun? (iii) How are GRB powered, and what are their high-energy behavior? (iv) How can high-energy $\gamma$-rays help probe the early universe and the cosmic evolution of high-energy sources to $z \geq 6$ ? and (v) What is the nature of Dark Matter? 

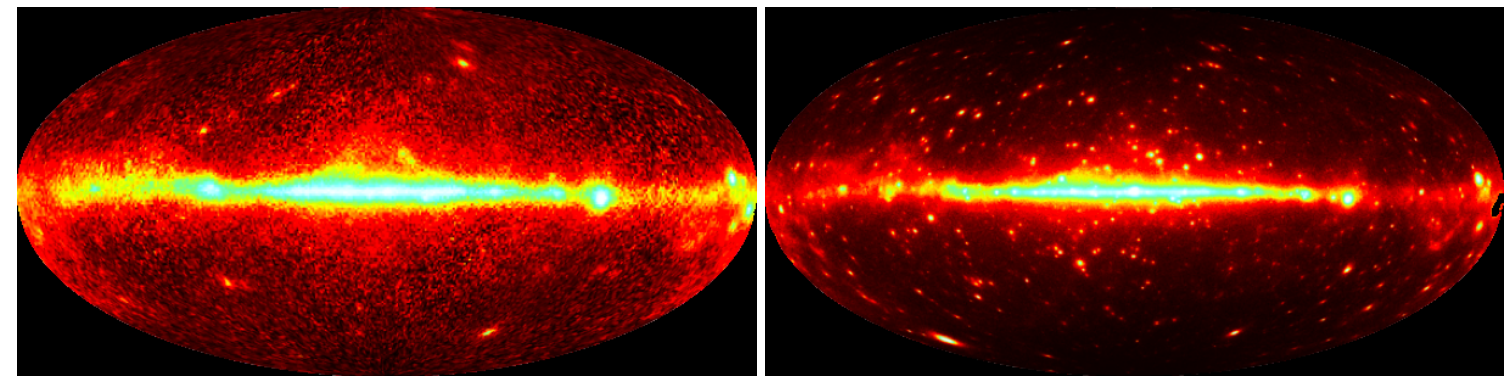

Figure 2: Comparison of the skymap for the full EGRET dataset (9 years) and for one year of simulated LAT data.
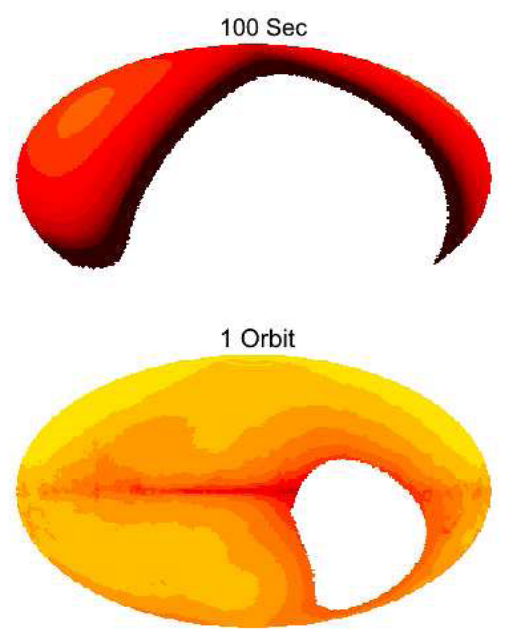
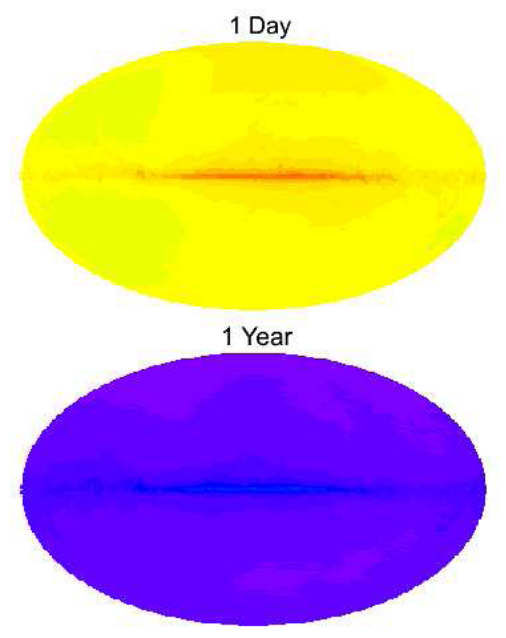

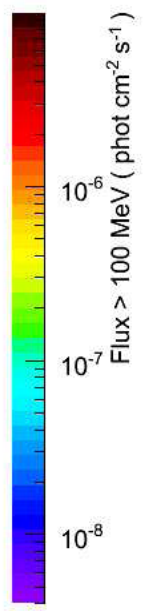

Figure 3: LAT source sensitivity for exposures on various timescales. Each map is an Aitoff projection in galactic coordinates. In standard sky-survey mode, nearly uniform exposure is achieved every 2 orbits, with every region viewed for $\sim 30$ min every 3 hours.

\section{Prospect for a Gamma-Ray Signal from Dark Matter with Fermi}

The Fermi LAT collaboration pursues complementary searches for Dark Matter, extensively discussed in [2]. Each strategy presents its own challenges and advantages, as summarized on table 3. In all cases, except perhaps the analysis of high-latitude dwarf galaxies, the diffuse emission from the Galaxy, mainly due to cosmic-ray interactions with the Galactic gas, is anticipated as the major source of background and of uncertainty in the extraction of a Dark Matter signal. In order to bracket this uncertainty in a reasonable though somewhat ad hoc fashion, Dark Matter sensitivity studies have been performed using two different Galactic diffuse background models, a conventional [9] model where local cosmic-ray abundance is assumed to be valid throughout the Galaxy, and an optimized [10] model where this constraint is relaxed in order to better fit EGRET data.

In order to study Dark Matter contribution from the Galactic halo, which is a very promising strategy [11], it is further necessary to completely remove part of the Galactic plane, either with a 


\begin{tabular}{lll}
\hline Search technique & Advantages & Challenges \\
\hline Galactic Center & good statistics & $\begin{array}{l}\text { source confusion, } \\
\text { galactic diffuse background }\end{array}$ \\
\hline Galactic Halo & very good statistics & galactic diffuse background \\
\hline Satellites & $\begin{array}{l}\text { low background, } \\
\text { good source id }\end{array}$ & low statistics \\
\hline Spectral Lines & $\begin{array}{l}\text { no astrophysical uncertainties, } \\
\text { good source id }\end{array}$ & low statistics \\
\hline Extragalactic & very good statistics & $\begin{array}{l}\text { astrophysics, } \\
\text { galactic diffuse background }\end{array}$ \\
\hline
\end{tabular}

Table 1: Overview of the various regions of interest for Dark Matter searches undertaken by the Fermi LAT collaboration. For more details see [2].

10 degree radial cut around the Galactic Center or with a 10 degree cut in the latitude. Results for a one year simulated dataset and for an NFW profile are shown in Figure 4.
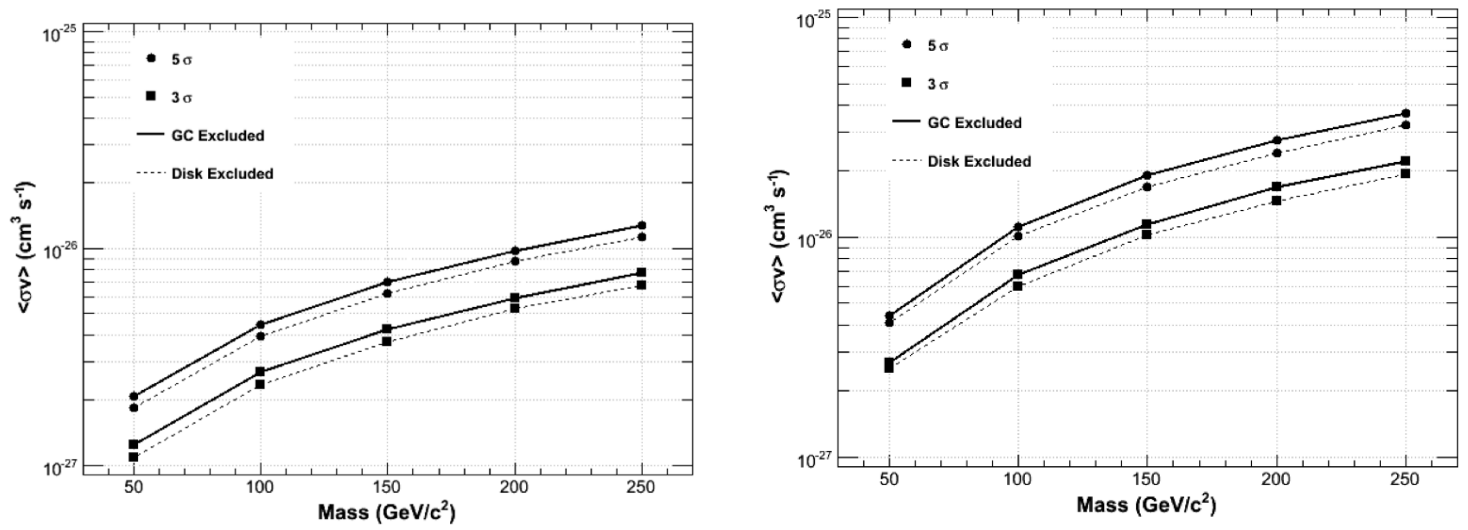

Figure 4: Fermi sensitivity to a Dark Matter signal from a NFW Galactic halo, after one year of observation, for the conventional (left) and optimized (right) models of Galactic diffuse emission. Results are shown for a 3 (dot) and 5 (square) sigma detection level, and for a disk (continuous line) or band (dashed line) removal of the Galactic foreground.

Furthermore, the analysis of the cosmological Dark Matter component in the isotropic diffuse gamma-ray emission requires complete removal of the Galactic diffuse background, as done for instance in [12]. Then, the dominant sources of uncertainty are the Dark Matter distribution over cosmological scales, and the extra-galactic isotropic diffuse component, for instance from unresolved blazars. Figure 5 (left) shows the continuum plus line spectrum for a 70 and $200 \mathrm{GeV}$ WIMP pair-annilating in the $b \bar{b}$ channel. The sensitivity plot for a one-year sky survey with Fermi is shown on the right of the same figure, varying assumptions on the extragalactic background from the optimistic case of a model for the population of unresolved blazars and the conservative assumption that the background is as measured by [12].

Finally, we direct the reader to $[15,16,17]$ for the specific cases of the Galactic Center, dwarf 

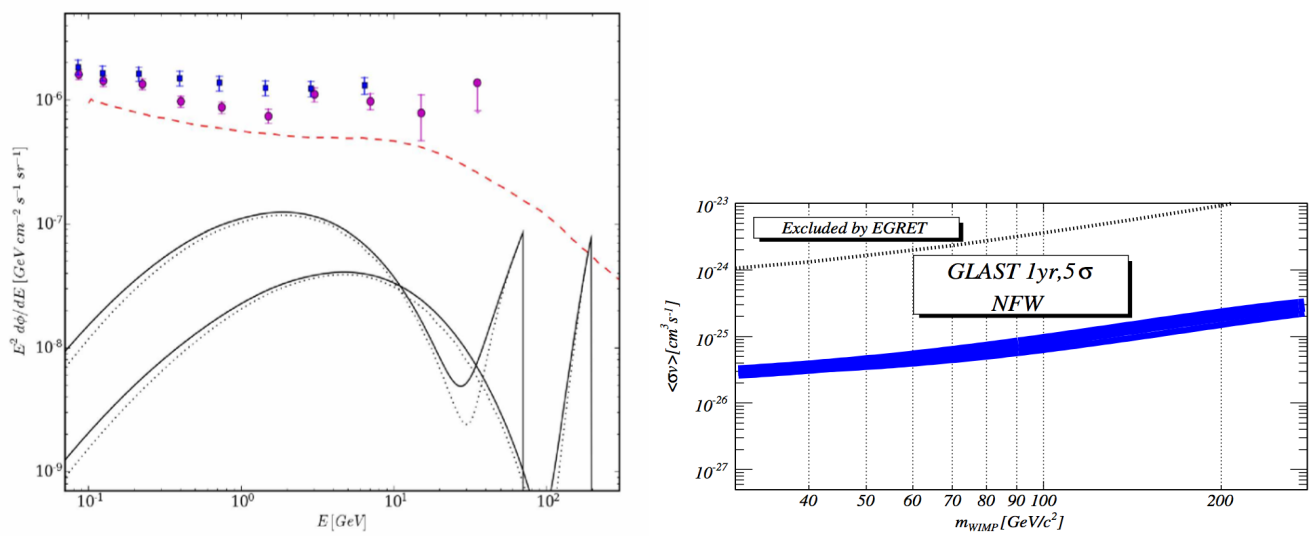

Figure 5: Left: Spectral shapes of the EGRET measurements of the extragalactic background, with [12] (dots) and [10] (squares) used for our conservative background, and [13] (dashed) for the the unresolved blazar model, and two examples of cosmological WIMPs with masses of 70 and $200 \mathrm{GeV}$. The dotted WIMP spectra are calculated with the absorption from [14]. Right: $5 \sigma$ exclusion curves for one year of Fermi simulated data. The lower edge of the shaded band corresponds to a background as predicted by the Blazar model presented in [13], and the upper bound corresponds to the conservative case, where the background flux is given by the analysis of EGRET data [12].

spheroidals, and line detection, respectively.

\section{Conclusions and Outlook}

Fermi is in routine science operations since August 11th, 2008. Its nominal sky-survey observation mode opens up the way to several complementary searches for Dark Matter signal in the gamma-ray sky, each presenting its own advantages and challenges. The establishment of a detection will require thorough understanding of the involved backgrounds and possibly joint observations with the ground based Cherenkov telescope experiments H.E.S.S., MAGIC, VERITAS and CANGAROO.

\section{References}

[1] G. Bertone, D. Hooper and J. Silk, Phys. Rept. 405 (2005) 279 [arXiv:hep-ph/0404175].

[2] E. A. Baltz et al., JCAP 0807 (2008) 013 [arXiv:0806.2911 [astro-ph]].

[3] A. Cesarini, F. Fucito, A. Lionetto, A. Morselli and P. Ullio, Astropart. Phys. 21 (2004) 267 [arXiv:astro-ph/0305075].

[4] T. Bringmann, L. Bergstrom and J. Edsjo, JHEP 0801 (2008) 049. See also T. Bringmann, these proceedings.

[5] M. Gustafsson, E. Lundstrom, L. Bergstrom and J. Edsjö, Phys. Rev. Lett. 99 (2007) 041301 [arXiv:astro-ph/0703512].

[6] W. B. Atwood [GLAST Collaboration], Nucl. Instrum. Meth. A 342, 302 (1994).

[7] P. F. Michelson [GLAST-LAT Collaboration], AIP Conf. Proc. 921, 8 (2007). 
[8] http://cossc.gsfc.nasa.gov/docs/cgro/cgro/egret.html

[9] A. W. Strong, I. V. Moskalenko and O. Reimer, Astrophys. J. 537, 763 (2000) [Erratum-ibid. 541, 1109 (2000)] [arXiv:astro-ph/9811296].

[10] A. W. Strong, I. V. Moskalenko and O. Reimer, Astrophys. J. 613, 962 (2004) [arXiv:astro-ph/0406254].

[11] C. Frenk, these proceedings.

[12] P. Sreekumar et al. [EGRET Collaboration], Astrophys. J. 494, 523 (1998) [arXiv:astro-ph/9709257].

[13] P. Ullio, L. Bergström, J. Edsjö and C. G. Lacey, Phys. Rev. D 66, 123502 (2002) [arXiv:astro-ph/0207125].

[14] F. W. Stecker, AIP Conf. Proc. 921, 237 (2007) [arXiv:astro-ph/0703505].

[15] V. Vitale, these proceedings.

[16] E. Nuss, these proceedings.

[17] T. Ylinen, these proceedings. 